\title{
Perfusion MR imaging for differentiation of benign and malignant meningiomas
}

\author{
Hao Zhang • Lars A. Rödiger • Tianzhen Shen • \\ Jingtao Miao $\cdot$ Matthijs Oudkerk
}

Received: 2 July 2007 / Accepted: 15 February 2008 / Published online: 1 April 2008

(C) The Author(s) 2008

\begin{abstract}
Introduction Our purpose was to determine whether perfusion MR imaging can be used to differentiate benign and malignant meningiomas on the basis of the differences in perfusion of tumor parenchyma and/or peritumoral edema. Methods A total of 33 patients with preoperative meningiomas ( 25 benign and 8 malignant) underwent conventional and dynamic susceptibility contrast perfusion MR imaging. Maximal relative cerebral blood volume (rCBV) and the corresponding relative mean time to enhance (rMTE) (relative to the contralateral normal white matter) in both tumor parenchyma and peritumoral edema were measured. The independent samples $t$-test was used to determine whether there was a statistically significant difference in the mean $\mathrm{rCBV}$ and $\mathrm{rMTE}$ ratios between benign and malignant meningiomas.

Results The mean maximal rCBV values of benign and malignant meningiomas were $7.16 \pm 4.08($ mean \pm SD) and $5.89 \pm 3.86$, respectively, in the parenchyma, and $1.05 \pm 0.96$ and $3.82 \pm 1.39$, respectively, in the peritumoral edema. The mean rMTE values were $1.16 \pm 0.24$ and $1.30 \pm 0.32$, respectively, in the parenchyma, and $0.91 \pm 0.25$ and $1.24 \pm 0.35$,
\end{abstract}

\footnotetext{
H. Zhang $(\bowtie) \cdot$ L. A. Rödiger $\cdot$ M. Oudkerk

Department of Radiology, University Medical Center Groningen, University of Groningen,

Groningen, The Netherlands

e-mail: zhanghao021@hotmail.com

H. Zhang $\cdot$ J. Miao

Department of Radiology, First People's Hospital,

Shanghai Jiaotong University,

Shanghai 200080, China

T. Shen

Department of Radiology, Huashan Hospital, Fudan University, Shanghai 200050, China
}

respectively, in the peritumoral edema. The differences in rCBV and rMTE values between benign and malignant meningiomas were not statistically significant $(P>0.05)$ in the parenchyma, but both were statistically significant $(P<0.05)$ in the peritumoral edema.

Conclusion Perfusion MR imaging can provide useful information on meningioma vascularity which is not available from conventional MRI. Measurement of maximal rCBV and corresponding rMTE values in the peritumoral edema is useful in the preoperative differentiation between benign and malignant meningiomas.

Keywords Meningioma. Cerebral neoplasm .

Magnetic resonance imaging · Perfusion-weighted imaging · Brain

\section{Introduction}

Meningiomas are the most common primary nonglial neoplasm, representing $15-20 \%$ of all primary brain tumors [1]. According to the classification of nervous system tumors of the World Health Organization (WHO) in 2000, there are three types of meningiomas based on malignant behavior: common benign (grade I), atypical (grade II), and malignant (grades III and IV). Grade I meningiomas have a recurrence rate of $6.9 \%$, and despite invasion of the adjacent bony structures, do not invade the brain parenchyma. Grade II meningiomas have a recurrence rate of $34.6 \%$, and show frequent mitosis and an increased nuclear-cytoplasmic ratio. Malignant meningiomas (grades III and IV) have a recurrence rate of $72.7 \%$, and show an even greater mitosis rate as well as necrosis and invasion of brain parenchyma [2].

Differentiating between malignant and benign meningiomas before surgery is important for both treatment planning 
and the prognosis appraisal. Although meningiomas do have some identifiable imaging features on conventional MR images, no special feature has been found to be reliable in predicting tumor grade. Sometimes, the aggressiveness of the tumor cannot be accurately characterized even on the basis of histopathological findings [3, 4]. Perfusion MR imaging reflects the characteristics of the regional blood supply, an important biological marker of tumor grade and prognosis. The purpose of this study was to evaluate the possible role of dynamic susceptibility contrast (DSC) perfusion MR imaging in grading of meningiomas. For the first time, the maximal relative cerebral blood volume (rCBV) (relative to the contralateral normal white matter) and corresponding relative mean time to enhance (rMTE) were measured in the tumor parenchyma and also in the peritumoral edema.

\section{Materials and methods}

\section{Patients}

Between January 2002 and December 2006, 33 patients (15 male, 18 female) with supratentorial meningiomas and peritumoral edema ( 25 benign and 8 malignant) were enrolled in this study. Their average age was 46 years, with a range of 17-63 years. All patients underwent gross total or subtotal resection of the tumor within 20 days of conventional and DSC perfusion MR imaging. All results were histopathologically confirmed.

\section{MRI protocol}

MR examinations were performed on a 1.5-T clinical system (Signa; GE Medical Systems; Milwaukee, WI). Sagittal and axial T1-weighted spin-echo (TR/TE 440/14 ms, matrix $256 \times 256$, section thickness $8 \mathrm{~mm}$, field of view $22 \mathrm{~cm}$ ), axial fluid attenuated inversion recovery (FLAIR) sequences (TR/TE 8002/126 ms) were performed before enhancement. Then $0.1 \mathrm{mmol} / \mathrm{kg}$ Gd-DTPA (Omniscan, GE Medical Systems) was administered intravenously with a power injector (Medrad, Indianola, PA) at a rate of $4 \mathrm{ml} / \mathrm{s}$, followed by a $20-\mathrm{ml}$ saline flush at the same rate. T2*-weighted dynamic susceptibility contrast-enhanced perfusion MR imaging was performed $5 \mathrm{~s}$ after the beginning of the injection using a gradient-echo echo-planar sequence with the following parameters: TR/TE $1800 / 40 \mathrm{~ms}$, number of excitations 1 , flip angle $60^{\circ}$, bandwidth 62.75 , matrix $128 \times$ 128 , number of sections 10 , section thickness $8 \mathrm{~mm}$ without spacing, 8-10 slices per acquisition, field of view $22 \mathrm{~cm}$, and acquisition time $1 \mathrm{~min} 38 \mathrm{~s}$. A series of 50 dynamic acquisitions were obtained for each section during the bolus injection of Gd-DTPA. Then, postcontrast sagittal and axial spin-echo T1-weighted sequences were performed using the same section positions and parameters as the corresponding precontrast sequences.

Data analysis

The images were analyzed using Functool software (ADW 4.0, GE Medical Systems, Milwaukee, WI). A dynamic perfusion technique with contrast medium demonstrates cerebral hemodynamics by analyzing changes in the signal intensities after administration of contrast material. During the first phase of passage of the contrast agent bolus, both $\mathrm{T} 2 *$ relaxation time and its equivalent $\mathrm{T} 2 *$ signal intensity decrease. The change in relaxation rate $(\Delta \mathrm{R} 2 *)$ can be calculated on the basis of signal intensity by means of the following equation: $\Delta \mathrm{R} 2 *=-\ln \left[\mathrm{S}(\mathrm{t}) / \mathrm{S}_{0}\right]$ echo time, where $\mathrm{S}(\mathrm{t})$ and $\mathrm{S}_{0}$ are the signal intensities at time $t$ and baseline. The recirculation and $\mathrm{T} 1$ shortening effects caused by contrast agent leakage can be reduced by fitting a gamma-variate function to the measured $\Delta \mathrm{R} 2 *$ curve. The area under the corrected contrast agent concentration-time curve is proportional to the $\mathrm{CBV}$ and does not yield an absolute value. It is necessary, therefore, to express $\mathrm{CBV}$ relative to that of a standard reference area, usually the contralateral white matter. We refer to this as relative CBV (rCBV). Since we cannot determine the arterial input function using this software, the mean time to enhance (MTE) in this study is related to the time to peak and to the width of the peak of the time signal curve; MTE is computed and displayed only for the images between the last preenhancement image and the first postenhancement image, equivalent to the bolus arrival time.

The steps to create perfusion maps have been described in detail previously by Knopp et al. [5]. The source images are first inspected for overall image quality and motion artifact. A single region of interest (ROI) is placed over the contralateral unaffected centrum semiovale white matter, and rCBV maps are generated to serve as road maps. CBV maps can then be calculated on a pixel-by-pixel basis and displayed as a gray-scale image. However, small but important variations in CBV are not always apparent from these maps. An alternative is to use a color overlay displayed on the raw image, in which the abnormal CBV values are often more apparent. A threshold for the color overlay at a CBV the same as that of the unaffected white matter was used in this study. In some peritumoral edema regions which had lower CBV than normal, the white matter showed as black in color and the maximal rCBV of these regions were determined mainly from the degree of signal intensity change (peak depth of the time-signal curve) of the respective ROIs. The degree of signal intensity change correlates well with $\mathrm{rCBV}$, so the region with the highest $\mathrm{rCBV}$ is easily identified. Furthermore, in lesions that are located close to major vascular structures, careful inspection of the signal intensity-time curve clearly 
indicates vessels that produce very large signal intensity changes. At least three circular ROIs, including at least 20 pixels each, were placed on the highest color levels of the solid part of the parenchyma and peritumoral edema of a tumor, and the maximal values were recorded. Peritumoral edema in this study was defined as areas of high signal on FLAIR images and within $1 \mathrm{~cm}$ of the outer enhancing tumor margin on postcontrast T1-weighted images. The same ROIs were also applied to MTE maps. For quantitative analysis, normal white matter within the contralateral hemisphere was used as the internal reference standard, and rCBV and rMTE values were calculated by dividing the maximal CBV and MTE of a tumor by that of the contralateral normal white matter.

Statistical analysis was performed with commercially available software (SPSS, version 14.0 for Windows; SPSS, Chicago, IL). The means of the maximum $\mathrm{rCBV}$ and the corresponding rMTE values of benign and malignant meningiomas were expressed as means \pm standard error of the mean. The differences between the means of benign and malignant meningiomas were compared using an independent-samples t-test. $P$ values less than 0.05 indicated a statistically significant difference.

\section{Results}

All tumors in this study were correctly diagnosed as meningiomas by evaluating the pre- and postenhancement images from conventional MRI sequences. No diagnosis of tumor malignancy or grading could be made on the basis of the conventional MR images. The parenchyma of more than half of the benign meningiomas $(n=16,64 \%)$ was isointense on T1-weighted MR images, and showed homogeneous enhancement after contrast medium administration with low signal of abnormal, tortuous tumor vessels in or surrounding the tumors. Peritumoral edema was most clearly seen on FLAIR images.

On the rCBV color maps, the parenchyma of all meningiomas showed uneven but higher blood volume than the surrounding tissues, and was represented as "hot" colors (red, orange and yellow). In all meningiomas, the
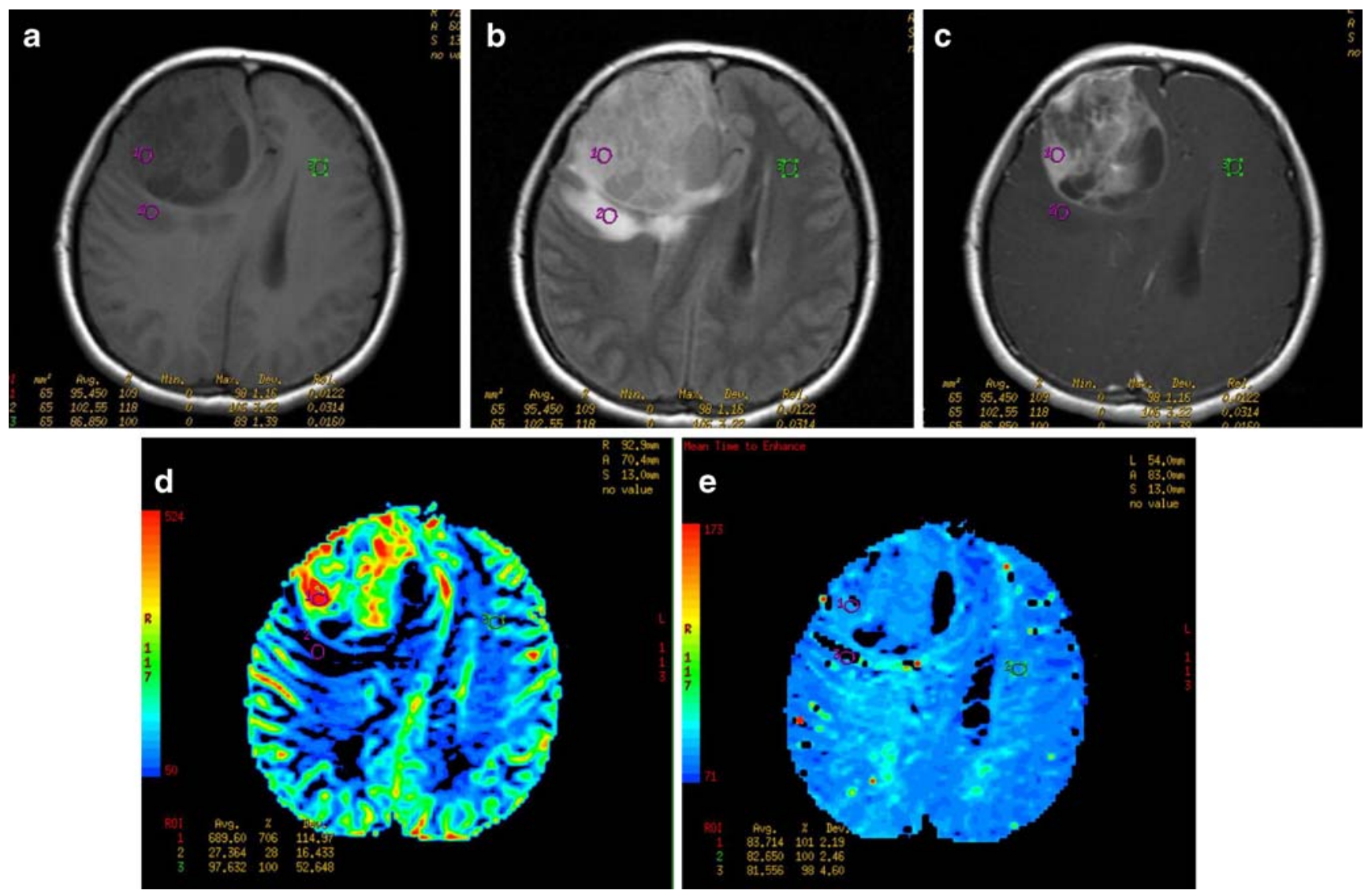

Fig. 1 Histopathologically confirmed benign meningioma (WHO grade I). a-c MR images. T1-weighted (a), FLAIR (b) and contrast-enhanced T1-weighted (c) images show an inhomogeneous mass lesion on the right convexity with little peritumoral edema and heterogeneous enhancement. d rCBV map. The "hot" colors (red) of ROI 1 in the tumor parenchyma indicates a higher rCBV than the normal white matter

(ROI 3, blue); the black color of ROI 2 in peritumoral edema indicates a lower rCBV than the normal white matter. The reference color scale is on the right side of the map. e rMTE map. The colors of the tumor parenchyma and peritumoral edema are similar to that of normal brain tissues; no clear interface can be seen 

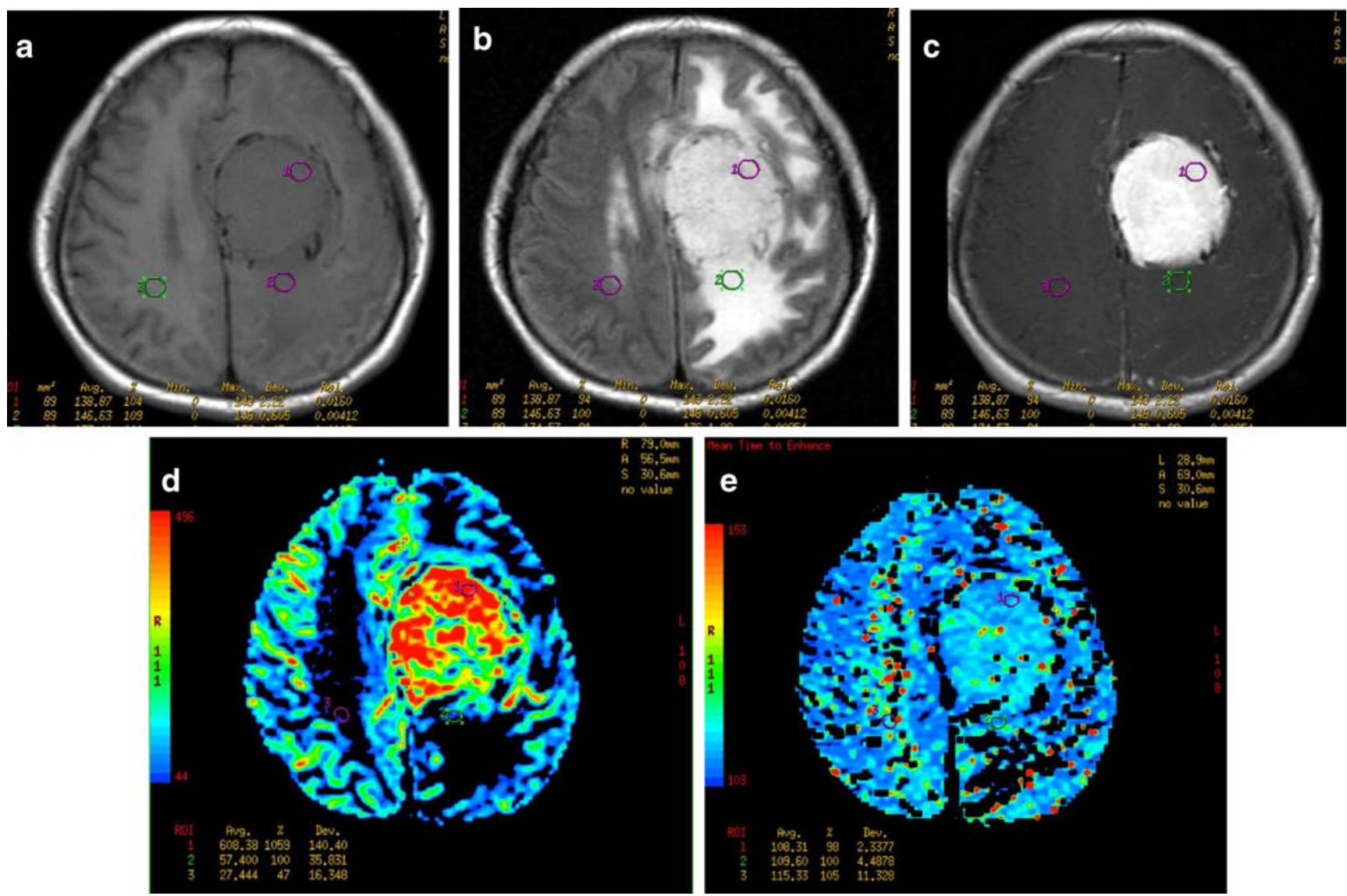

Fig. 2 Histopathologically confirmed malignant meningioma (WHO grade III). a-c MR images. T1-weighted (a), FLAIR (b) and contrastenhanced T1-weighted (c) images show a mass with nearly isointense parenchyma accompanied by widespread peritumoral brain edema and strong homogeneous enhancement. d rCBV map. The red color of ROI 1 in the tumor parenchyma indicates a higher rCBV than the

blood volume of peritumoral edema was lower than in tumor parenchyma. Some marginal parts of malignant meningiomas were blurred on the rCBV maps and showed no clear interface with the surrounding brain tissues. The areas of maximal blood volume in the peritumoral edema of malignant meningiomas were represented as slightly "hotter" or the same colors (green and blue) compared to normal white matter (blue) on the rCBV color maps. While both the tumor parenchyma and peritumoral edema of malignant meningiomas were represented as slightly "hot- surrounding brain tissues; the green and blue colors of ROI 2 in the peritumoral edema are also "hotter" than ROI 3 (dark) in the normal white matter. The reference color scale is on the right side of the map. e MTE map. Most of the tumor parenchyma shows slightly "hotter" colors than the normal brain tissue, reflecting a prolonged rMTE

ter" colors than areas of normal brain tissues on rMTE color maps, the tumor parenchyma and peritumoral edema of benign meningiomas showed similar colors to normal brain tissue on rMTE color maps. The interfaces between tumor parenchyma and peritumoral edema of benign meningiomas were blurred on rMTE color maps (Figs. 1 and 2).

The mean maximal rCBV values and corresponding rMTE values of tumor parenchyma and peritumoral edema are shown in Table 1 with the statistical results derived from comparing the mean values between benign and malignant meningiomas.

Table 1 Comparison of rCBV and rMTE values of benign and malignant meningiomas. The values presented are relative values in comparison with the contralateral white matter (assumed to have a value 1.00)

\begin{tabular}{|c|c|c|c|c|}
\hline \multirow[t]{2}{*}{ Meningioma } & \multicolumn{2}{|l|}{$\mathrm{rCBV}$} & \multicolumn{2}{|l|}{ rMTE } \\
\hline & Parenchyma & Peritumoral edema & Parenchyma & Peritumoral edema \\
\hline Benign $(n=25)$ & $7.16 \pm 4.08$ & $1.05 \pm 3.86$ & $1.16 \pm 0.24$ & $0.91 \pm 0.25$ \\
\hline Malignant $(\mathrm{n}=8)$ & $5.89 \pm 3.86$ & $3.28 \pm 1.39$ & $1.30 \pm 0.32$ & $1.24 \pm 0.35$ \\
\hline$P$ value $^{\mathrm{a}}$ & 0.078 & 0.002 & 0.278 & 0.032 \\
\hline
\end{tabular}

${ }^{\text {a }}$ Independent samples $t$-test. $P$ values $<0.05$ indicate a statistically significant difference 


\section{Discussion}

The distinction between benign and malignant meningiomas is clinically relevant because of their different recurrence rate [2]. Differentiating between benign and malignant meningioma before surgery could be helpful in the planning of surgical resection and adjunctive radiation therapy. The characteristics of meningiomas demonstrated on images derived from conventional MR sequences cannot always be used to reliably differentiate between benign and malignant tumors. In our study of 33 patients, all tumors were diagnosed correctly as meningiomas using conventional MR sequences. Nevertheless, distinguishing between benign and malignant meningiomas can be difficult if not impossible with conventional MR imaging sequences.

Determining CBV from DSC perfusion MR imaging data has previously been shown to be useful in differentiating gliomas of different grades [5,6]. The most widely used DSC perfusion MR imaging technique is based on the intravascular indicator dilution theory of Zierler [7] which states that, in the absence of recirculation and contrast material leakage, $\mathrm{CBV}$ is proportional to the area under the contrast agent concentration-time curve and does not yield an absolute measurement. For the latter it is necessary to express $\mathrm{CBV}$ relative to that of a standard reference area, usually the contralateral white matter. Nevertheless, the above-mentioned assumptions are violated in meningiomas, because they lack a true blood-brain barrier. Several methods for correcting for the under- or overestimation of rCBV measurements from DSC imaging in regions of microvascular leakage and for compensating for the $\mathrm{T} 1$ shortening effects caused by contrast leakage into the tumoral interstitial space have been studied, but none is considered a standard reference method [8-10].

Previous attempts to assess the grade of meningiomas using DSC perfusion MR with determination of the rCBV of the tumor parenchyma have not been successful. Zhu et al. [11] found no correlation between $\mathrm{rCBV}$ and tumor grade in 2 malignant and 12 benign meningiomas. In another study Yang et al. [8] found no significant difference between the rCBV values of 7 atypical and 15 benign meningiomas. These findings are consistent with our results. In our study, the mean $\mathrm{rCBV}$ value derived from the maximal blood volume regions in the parenchyma of benign meningiomas was higher than that of malignant meningiomas, but the difference between the two groups showed no statistical significance. The means of the MTE values in the parenchyma of both benign and malignant meningiomas were longer than those of normal brain tissue but there was no statistically significant difference between benign and malignant meningiomas. These findings could be attributed to the immature and tortuous tumor vessels which could result in an increased leakage of contrast agent from vessels into the extravascular space and deceleration of blood flow in the tumor parenchyma.

The notable and original results of this study were that the mean maximal $\mathrm{rCBV}$ and corresponding rMTE values of peritumoral edema of malignant meningiomas were greater than those of benign meningiomas and significant differences were found between the two groups $(P<0.05)$. The increase in $\mathrm{rCBV}$ in the peritumoral edema of malignant meningiomas might be attributed to tumor invasion and angiogenesis in the adjacent brain tissue [12]. Previous studies focusing on the pathological and molecular characteristics of meningiomas have shown that FLT1- and VEGF-positive cells are increased in the endothelium of intratumoral vessels and are associated with microvascular proliferations in peritumoral brain tissue of anaplastic meningiomas [13]. We consider that the different transit times between benign and malignant meningiomas in our study group was due to a multifactorial effect including tumor size, vascular compression, capillary permeability and vessel tortuosity, which have been evaluated in other studies $[14,15]$. To our knowledge, no previous studies have examined the microcirculation in the peritumoral edema of meningiomas by means of perfusionweighted MR imaging. In this study, the number of patients was limited and physiopathological details of the areas of peritumoral edema were not available for comparison with the perfusion MR findings in order to prove the assumptions made. Studies with larger sample sizes and more detailed evaluation of the physiopathology of the peritumoral edema of meningiomas of different grade are needed.

\section{Conclusion}

DSC perfusion MR imaging can provide critical information on the vascularity of meningiomas which is not available with conventional MR imaging. Determination of maximal $\mathrm{rCBV}$ and corresponding rMTE values in the peritumoral edema is useful in the preoperative differentiation between benign and malignant meningiomas.

Conflict of interest statement We declare that we have no conflict of interest.

Open Access This article is distributed under the terms of the Creative Commons Attribution Noncommercial License which permits any noncommercial use, distribution, and reproduction in any medium, provided the original author(s) and source are credited.

\section{References}

1. Sheporaitis L, Osborn A, Smirniotopoulos J, Clunie DA, Howieson J, D'Agostino AN (1992) Radiologic-pathologic correlation: intracranial meningioma. AJNR Am J Neuroradiol 13:29-37 
2. Kleihues P, Louis DN, Scheithauer BW, Rorke LB, Reifenberger G, Burger PC, Cavenee WK (2002) The WHO classification of tumors of the nervous system. J Neuropathol Exp Neurol 61: 215-225

3. Brainard JA, Prayson RA, Barnett GH (1997) Frozen section evaluation of stereotactic brain biopsies: diagnostic yield at the stereotactic target position in 188 cases. Arch Pathol Lab Med 121:481-484

4. Hall WA (1998) The safety and efficacy of stereotactic biopsy for intracranial lesions. Cancer 82:1749-1755

5. Knopp EA, Cha S, Johnson G, Mazumdar A, Golfonos JG, Zagzag D, Kelly PJ, Kricheff II (1999) Glial neoplasms: dynamic contrast-enhanced T2*-weighted MR imaging. Radiology 211: 791-798

6. Aronen HJ, Gazit IE, Louis DN (1994) Cerebral blood volume maps of gliomas: comparison with tumor grade and histologic findings. Radiology 191:41-51

7. Zierler KL (1962) Theoretical basis of indicator-dilution methods for measuring flow and volume. Circ Res 10:393-407

8. Yang S, Law M, Zagzag D, Wu HH, Cha S, Golfinos JG, Knopp EA, Johnson G (2003) Dynamic contrast-enhanced perfusion MR imaging measurements of endothelial permeability: differentiation between atypical and typical meningiomas. AJNR Am J Neuroradiol 24:1554-1559
9. Cha S (2006) Update on brain tumor imaging: from anatomy to physiology. AJNR Am J Neuroradiol 27:475-487

10. Spampinato MV, Wooten C, Dorlon M et al (2006) Comparison of first-pass and second-bolus dynamic susceptibility perfusion MRI in brain tumors. Neuroradiology 48:867-874

11. Zhu F, Zhou Y, Wang C et al (2002) Perfusion MRI evaluation of correlating perfusion constants with histologic findings in meningiomas. Proceedings of the Annual Meeting of the International Society for Magnetic Resonance in Medicine. Berkeley, CA

12. Arai M, Kashihara K, Kaizaki Y (2006) Enhancing gliotic cyst wall with microvascular proliferation adjacent to a meningioma. $\mathrm{J}$ Clin Neurosci 13:136-139

13. Yoshioka H, Hama S, Taniguchi E, Sugiyama K, Arita K, Kurisu K (1999) Peritumoral brain edema associated with meningioma: influence of vascular endothelial growth factor expression and vascular blood supply. Cancer 85:936-944

14. Nakano T, Asanoa K, Miura H, Itoh S, Suzuki S (2002) Meningiomas with brain edema: radiological characteristics on MRI and review of the literature. Clin Imaging 26:243-249

15. Cha S, Yang L, Johnson G, Lai A, Chen MH, Tihan T, Wendland M, Dillon WP (2006) Comparison of microvascular permeability measurements, K(trans), determined with conventional steady-state T1weighted and first-pass $\mathrm{T} 2 *$-weighted $\mathrm{MR}$ imaging methods in gliomas and meningiomas. AJNR Am J Neuroradiol 27:409-417 